\title{
Brain Activation and Psychophysiologic Interaction in Association with a Phonological Working Memory Task
}

\author{
Ahmad Nazlim Yusoff ${ }^{1}$, Hanani Abdul Manan ${ }^{1}$, Siti Zamratol-Mai Sarah Mukari ${ }^{2}$, Khairiah Abdul Hamid ${ }^{1,3} \&$ \\ Elizabeth A. Franz ${ }^{4}$ \\ ${ }^{1}$ School of Diagnostic and Applied Health Sciences, Universiti Kebangsaan Malaysia, Kuala Lumpur, Malaysia \\ ${ }^{2}$ School of Rehabilitation Sciences, Universiti Kebangsaan Malaysia, Kuala Lumpur, Malaysia \\ ${ }^{3}$ School of Health Sciences, KPJ Healthcare University College, Negri Sembilan, Malaysia \\ ${ }^{4}$ Department of Psychology and fMRIotago, University of Otago, Dunedin, New Zealand \\ Correspondence: Ahmad Nazlim Yusoff, Diagnostic Imaging \& Radiotherapy Program, School of Diagnostic \\ and Applied Health Sciences, Faculty of Health Sciences, Universiti Kebangsaan Malaysia, Jalan Raja Muda \\ Abdul Aziz, 50300 Kuala Lumpur, Malaysia. Tel: 603-2687-8084. E-mail: nazlimtrw@ukm.edu.my
}

$\begin{array}{lr}\text { Received: June 10, } 2014 & \text { Accepted: June 16, } 2014 \quad \text { Online Published: August 16, } 2014 \\ \text { doi:10.5539/mas.v8n5p97 } & \text { URL: http://dx.doi.org/10.5539/mas.v8n5p97 }\end{array}$

The research is financed by the UKM-GUP-SK-07-20-205 research grant.

\begin{abstract}
Brain activation within, and psychophysiologic interaction between, significantly activated regions in the brain obtained from a phonological working memory experiment on a single participant were studied. Given that working memory and speech processing are key functions of human behaviour, this type of investigation is of fundamental importance to our understanding of brain-behaviour relationships. The study objectives were to determine the areas that respond significantly to a phonological working memory task and to investigate the influence of babble noise on their activation and the psychophysiologic interactions (PPI) between the source region and those activated areas. Three conditions were used during functional magnetic resonance imaging (fMRI) scans which were working memory in quiet (WMQ), working memory in noise (WMN) and listening to babble noise $(\mathrm{N})$. More voxels are activated in the right temporal lobe than in the left during $\mathrm{N}$ condition due to the non-speech stimulus. However, a higher mean stimulus efficacy $(\varepsilon)$ of the point of maximum intensity in the left temporal lobe causes its signal intensity to be higher than in the right temporal lobe. Both the WMQ and WMN conditions resulted in similar activated regions in the brain but with a higher number of activated voxels (NOV) during WMQ for the right hemispheric areas in association with the working memory task. This is due to the sensitivity of those regions in perceiving and performing the phonological working memory task in quiet to a level that actually exceeds the activation enhancement commonly associated with the performance of working memory task in noise. This is supported by the PPI results that performing the working memory task is less influenced by noise for that particular brain region.
\end{abstract}

Keywords: functional magnetic resonance imaging, statistical parametric mapping, working memory, brain activation, psychophysiologic interaction

\section{Introduction}

In spite of extensive research conducted on human brain processes, our knowledge about specific functions associated with storing and manipulating information (i.e., working memory and reasoning), and how background noise influences this special ability, is still in a rapid growth period (Brzezicka et al. 2011; Gathercole 1999; Karlsgodt et al. 2005; Toepper et al. 2010). Working memory is different from short term memory. The distinction between the two is that the latter does not involve the processing of a cognitive task such as mental calculation or decision making. In contrast to short-term memory, working memory can be defined as the capacity of the brain to retain information for a brief period of time for the purpose of manipulating and processing of cognitive information. A novel model on working memory was proposed by Baddeley and Hitch (1974) and has become a gold standard since then. The model comprises a three-component system consisting of the central executive, phonological loop and visuospatial sketchpad. Twenty two years after 
its original development, the fourth component was introduced into the original model (Baddeley 1996) and was named the episodic buffer. While the present study focuses on specific processes of working memory of speech-related information (under the influence of background noise), the interested reader can refer to some exceptional reviews on the development of working memory models and theories as applied across a vast number of studies (Baddeley 2002; Gathercole 1999).

Known brain processes involved in working memory processing include cortical regions of the posterior parietal cortex, Broca's area, premotor and supplementary motor areas (Brodmann Area (BA) 40, 44 and 6) in the left hemisphere for phonological loop function. In the right hemisphere are the right inferior prefrontal cortex, right anterior occipital cortex, right posterior parietal cortex and right premotor cortex (BA 47, 19, 40 and 6) for the visuospatial sketchpad. Bilateral prefrontal cortex (BA 9, 10, 44, 45 and 46) is also known to be associated with central executive function (Gathercole 1999).

One interesting sub-cortical region involved in working memory processing is the hippocampus, a paired structure located inside the medial temporal lobe beneath the cortical surface. The hippocampus has been found to mediate working memory processing such as encoding the change in locations (Toepper et al. 2010), maintenance and retrieval (Karlsgodt et al. 2005) in addition to its known role in spatial learning and long term memory. In an fMRI study using a Corsi Block-Tapping test (CBT), it has also been found (Toepper et al. 2010) that other cortical and subcortical areas were activated during working memory which included the superior frontal gyrus, precentral gyrus, superior parietal lobule, inferior parietal lobule, middle occipital gyrus, inferior occipital gyrus, insula, putamen, caudate and cerebellum.

The present study, which investigates the effects of babble noise on working memory processing of speech stimuli is based on the foundation that working memory plays an important role in maintaining, manipulating, processing and storing information during cognitive processes. Brain activation that results during the execution of a task will peak normally between 5 to $10 \mathrm{~s}$ after stimulus delivery (Amaro \& Barker 2006; Belin et al. 1999; Bernal \& Altman 2001; Hall et al. 1999; Hall et al. 2000) with the dispersion of the peak around 2 to $3 \mathrm{~s}$. In order for this brief response of the brain to be captured without being interfered by the scanner noise, a short-acquisition time (TA) of $2 \mathrm{~s}$ with a long-repetition time (TR) of $16 \mathrm{~s}$ was applied in a sparse temporal sampling functional magnetic resonance imaging (STS-fMRI) protocol (Gaab et al. 2003). The data were analysed using Statistical Parametric Mapping (SPM8) from which the spatial and height extent of activation in the brain were compared and contrasted between the execution of working memory task in quiet and the same task in a noisy background. Another objective of this work is to investigate and measure the psychophysiologic interaction (PPI) between the input area and the areas that are activated when the participant performed the working memory task. PPI is defined as the responses in an activated cortical area in terms of an interaction between the influence of another activated area and an experimental factor (Friston et al. 1997). In this work, the experimental factor is the babble noise itself. What this study aimed to discover is whether babble noise has some measurable effects on the slope of a regression line when the response in one region is regressed on the activity in the second region.

This study was conducted on a single male participant. Thus, the inferences made about the brain activation (data) evoked by the execution of working memory task in different environments are only valid for this particular participant with a variance given by $\sigma_{w}{ }^{2} / n$. The subscript $w$ denotes within participant and $n$ is the number of measurements. The error variance was estimated on a scan-by-scan basis with the assumption that each scan represents an independent observation with no serial correlation. Assessing brain activations obtained from a single participant has the advantage that they exhibit the true effects of that particular participant at a relatively high threshold and the effects observed are not influenced by between participants variability which sometimes can be cascaded down into the average effects of group activation. On the other hand, inferences about the population are not possible given there is no test of reproducibility of the data. However, this study will serve as a baseline for future multiple participant studies in a similar context in the attempt to gain a better understanding of the brain functional and structural cortical connections during the execution of working memory and how such processes are mediated by experimental factors such as noise.

\section{Methods}

\subsection{Participant}

A phonological working memory experiment was conducted on a single right-handed Malay male participant using functional magnetic resonance imaging (fMRI). The participant is a 27 -year old native Malay male speaker with no reported history of psychiatric or neurological disorder and no current use of any psychoactive medications. The participant had no hearing impairment and no history of long time exposure to loud noise to be 
inappropriate for auditory stimulus presentation. The participant's hearing levels for both ears are not greater than $30 \mathrm{~dB}(\mathrm{HL})$ in the frequency range of $250-8,000 \mathrm{~Hz}$. The participant was confirmed to be right-handed with laterality index of 82.14 (in the range of $5^{\text {th }}$ right). The participant agreed to participate by filling up the informed consent and screening forms and signing them, after full explanation of the nature and risks of the research, as required by the Institutional Ethics Committee (IEC) (Reference no: FF-205-2006). The participant was then interviewed on his health condition prior to the scanning session and tested for middle ear conditions (Tympanometer Model Grason Stadler Inc. GSI33) and hearing level (Pure tone audiometer Model Grason Stadler Inc. GSI61) in the frequency range of $250 \mathrm{~Hz}$ to $8,000 \mathrm{~Hz}$, by a qualified Audiologist. Prior to the fMRI scans, the participant's handedness was tested using the Edinburgh Handedness Inventory (Oldfield 1971). The participant was told not to move his head during the scan and was instructed to attend and respond to the given stimulus accordingly.

\subsection{Working Memory Paradigm}

A sparse temporal sampling functional magnetic resonance imaging (STS - fMRI) was used in this study due to its relatively high sensitivity in detecting brain activation and in order to avoid the interference of the scanner sound with the auditory paradigm (Mueller et al. 2011). There were altogether 122 functional volumes in the whole imaging session. The first two volumes were dummies and the images were not acquired. Each functional volume consisted of 32 axial slices that were acquired in 2-s acquisition time (TA) (one image slice in $62.5 \mathrm{~ms}$ ) with an inter-scan interval (TR) of $16 \mathrm{~s}$. The imaging time for the whole functional scan is $1,920 \mathrm{~s}$ (32 minutes) which produces $120 \times 32=3,840$ images in total.

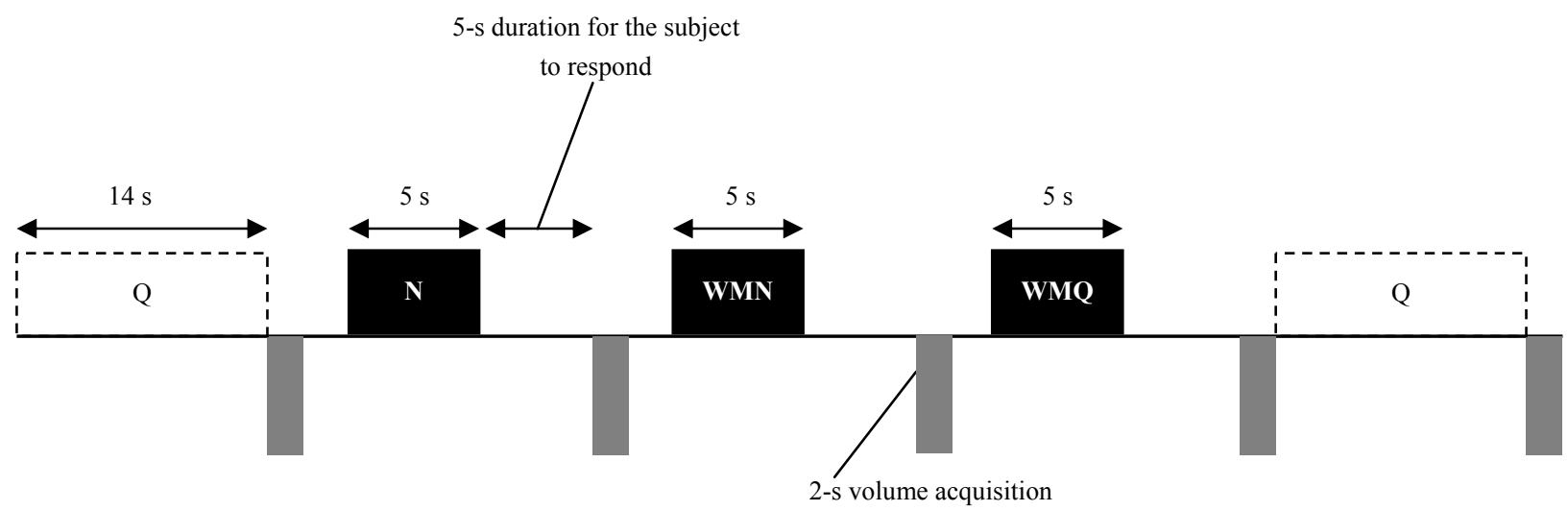

Figure 1. Schematic illustration of a cycle of the sparse temporal sampling used in this study; $\mathrm{Q}=$ quiet condition, $\mathrm{N}=$ babble noise condition, WMN = working memory performed with the presence of babble noise, and WMQ $=$ working memory performed in quiet

The working memory stimulus was presented for $5 \mathrm{~s}$ during the 14-s gap of scanner silence, at the 4th second after each successive acquisition, using a digital playback system. Fig. 1 shows a schematic illustration of stimulus presentation during the STS. The stimulus consisted of a series of 5 Malay nouns (e.g, buku, kayu, kain, bola, meja) which were verbally presented at random. The participant was instructed to listen to the given words, memorise and then verbally 'rephrase' all the words backwardly immediately after hearing the last word. The participant was given $5 \mathrm{~s}$ to respond to the given stimuli and was requested to remain focused throughout the entire scanning session. Three conditions were used in this study; working memory in quiet (WMQ), working memory in babble noise (WMN) and listening to babble noise (N). In WMQ, the participant was required to rephrase backward all the words that he heard without being distracted. Similar stimulus presentation was used for WMN but the words were embedded in babble noise (Stimulus-to-noise ratio: $\mathrm{SNR}=5 \mathrm{~dB}$ ) as distractor. The babble noise was recorded in a session where five multi-talkers read the same paragraph of text in an unsynchronised tempo. A total of 20 trials for each of the three N, WMN and WMQ conditions were alternately presented to the participant. A number of 20 functional image volumes were also acquired during which the participant listened only to the noise $(\mathrm{N})$. Additionally, 60 volume acquisitions during the quiet condition (Q) were also conducted. All stimuli were presented binaurally at the intensity level of $70 \mathrm{~dB}$ via a headphone which was connected to a digital playback system. The stimuli were transmitted to both ears via air tubes. 


\section{3 fMRI Scans}

Functional magnetic resonance imaging (fMRI) scans were performed using a 1.5 tesla magnetic resonance imaging (MRI) system (Siemens Magnetom Avanto) equipped with functional imaging option, echo planar imaging capabilities and radiofrequency (RF) head coil used for signal transmission and reception. Gradient Echo - Echo Planar Imaging (GRE-EPI) pulse sequence with the following parameters were applied to produce high resolution T2*-weighted images: Repetition time $(\mathrm{TR})=16,000 \mathrm{~ms}$, acquisition time $(\mathrm{TA})=2,000 \mathrm{~ms}$, echo time $(\mathrm{TE})=50 \mathrm{~ms}$, field of view $(\mathrm{FOV})=192 \times 192 \mathrm{~mm}$, flip angle $(\alpha)=90^{\circ}$, voxel size $=3 \mathrm{~mm}^{3}$ isotropic, slice thickness $=3 \mathrm{~mm}$. In addition, anatomical images of the entire brain were obtained using a T1-weighted multiplanar reconstruction (MPR) pulse sequence with the following parameters: TR $=1620 \mathrm{~ms}, \mathrm{FOV}=250 \times$ $250 \mathrm{~mm}, \alpha=90^{\circ}$, voxel size $=3 \mathrm{~mm}^{3}$ isotropic and slice thickness $=1 \mathrm{~mm}$.

\subsection{Behavioral Study}

The participant's performance in both WMQ and WMN conditions was scored as the percentage of the series of words that have been correctly rephrased. A qualitative interpretation on the participant's performance during $\mathrm{WMQ}$ and $\mathrm{WMN}$ is made in relation to the fMRI results.

\subsection{Image Processing}

The first two scans were discarded to eliminate the effects of magnetic saturation. The fMRI data were analysed using MATLAB 7.4 - R2008a (Mathworks Inc. MA, USA) and Statistical Parametric Mapping (SPM8) (Functional Imaging Laboratory, Wellcome Department of Imaging Neuroscience, Institute of Neurology, University College of London) programming software. The participant's structural T1 and functional T2 weighted images were normalised based on the standard templates produced by the Montreal Neurological Institute (MNI). Conventional fixed-effects (FFX) and conjunction analyses based on the general linear model (GLM) were used to generate brain activation in the regions of interest using the $T$-statistic for each voxel at $\alpha=$ 0.05. Due to the spatial correlation that is cascaded down from the smoothed functional images, the brain activations obtained from GLM were inferred at corrected significant level $\left(\alpha_{\mathrm{FWE}}\right)$ of 0.05 using random field theory (Friston et al. 1996). The fact that this study deals with volume or family of voxel statistics, the related risk of error is known as the family-wise error (FWE) rate which is defined as the likelihood that a particular family of voxel could have arisen by chance if the null hypothesis of no effects (activation) is rejected. The cortical regions which are found to be significantly activated during the phonological working memory task are defined using the Anatomy toolbox (Eickhoff et al. 2005) at $\alpha_{\mathrm{FWE}}=0.05$, corrected for multiple comparisons.

The hemodynamic properties of the area of interest were also estimated using SPM to determine the mean efficacy of the stimulus $(\varepsilon)$ in generating BOLD responses (Friston et al. 2000). This is achieved by extracting the hemodynamic parameters from a 4-mm radius sphere centered at the point of maximum intensity of each respective area. Spearman's correlation analysis was then conducted to determine the existence of any relationship between the effect size ( $t$ value) and the mean stimulus efficacy at $\alpha=0.05$ (95\% CI) for each particular area of interest.

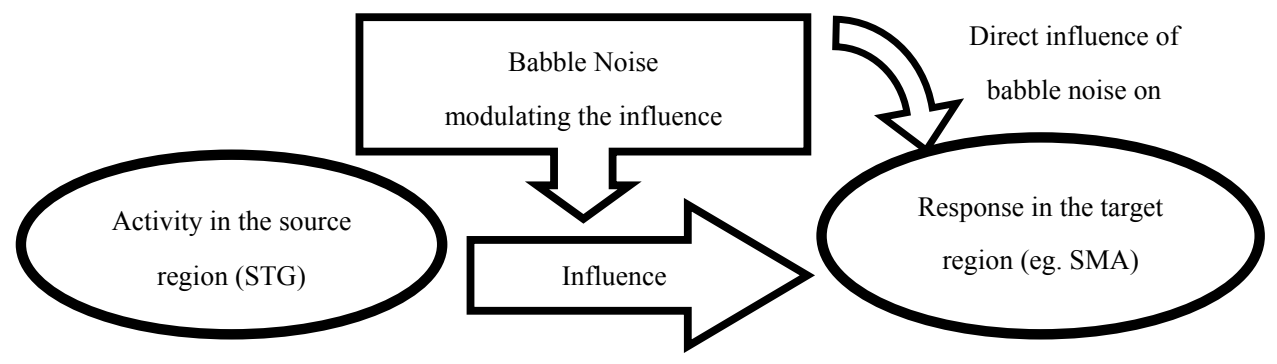

Figure 2. PPI model used in psychophysiologic interaction analysis

Psycophysiologic interaction (PPI) was used in this study to investigate the responses in one region of interest (ROI), or source region, in terms of an interaction between the influence of another ROI, target regions, and the babble noise (Friston et al. 1997). A 4-mm radius sphere with the point of maximum intensity as the centre was drawn for each of the source and target regions. The PPI responses for the target regions were then plotted as a function of the activity in the source region, assumed to be interacting with an experimental factor (babble noise), which in turn influences the response in the target area. The psychophysiologic interaction can be understood in 
terms of looking for a significant difference in the regression slope of the activity in the source region on response in a target region with and without the interaction with the experimental factor. Illustration of the PPI model used in this study is shown in Fig. 2 and will be explained in the Discussion section.

\section{Results}

\subsection{Behavioural Results}

The results obtained from the behavioural data indicated that $90 \%(18 / 20)$ of the participant's responses were correct in the noisy background as compared to $85 \%(17 / 20)$ in quiet. However, the significance of the difference was not able to be determined from these single-participant data.

\subsection{Brain Activation Characteristics}

Fig. 3 shows the brain areas that are activated when the participant was listening to babble noise. Two activation clusters survived the spatial threshold of 5 voxels and the height threshold of $t=5.21$ ( $p_{\text {FWE }}<0.05$ for each voxel, corrected for multiple comparisons). Three local maxima more than $8.0 \mathrm{~mm}$ apart were observed in each cluster. The coordinates of the first two maxima in one hemisphere are the mirror image of the ones in the other hemisphere. However, the $x$ and $z$ coordinates for the third maxima in the two hemispheres are almost the same but differ largely in the $y$ direction. Some important data characterising the brain activation in Fig. 3 are tabulated in Table 1.

(a)
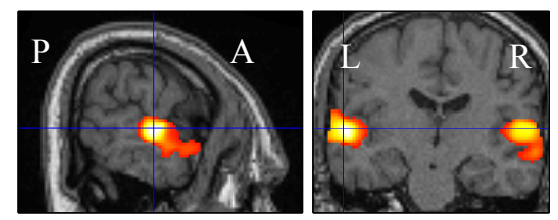

(b)

(c)

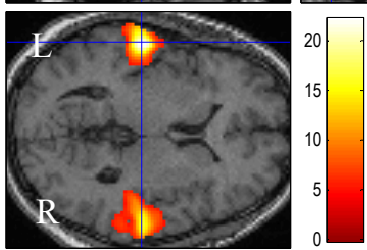

Figure 3. Brain activation resulting from listening to babble noise, overlaid onto the subject's (a) sagittal, (b) coronal and (c) axial normalised T1-weighted images $(\mathrm{P}=$ Posterior, $\mathrm{A}=$ Anterior, $\mathrm{L}=\mathrm{Left}, \mathrm{R}=\mathrm{Right})$. The $t$-values for the activated voxels are scaled in colors (red to white) as shown

Table 1. Characteristics of brain activation shown in Fig. 3

\begin{tabular}{llllllllll}
\hline Cluster & $\begin{array}{l}\text { Local } \\
\text { maximum }\end{array}$ & Region & $K_{\mathrm{E}}$ & $x$ & $y$ & $z$ & $t$-value & $p_{\text {FWE }}$ & $\varepsilon / \mathrm{s}^{-1}$ \\
\hline 1 & 1 & Left STG & 1,489 & -58 & -22 & 6 & 22.21 & $<0.05^{*}$ & 3.65 \\
& 2 & Left MTG & & -64 & -14 & -6 & 12.97 & $<0.05^{*}$ & 1.32 \\
& 3 & Left MTG & & -68 & -34 & 12 & 12.35 & $<0.05^{*}$ & 1.26 \\
& & & & & & & & & \\
2 & 1 & Right STG & 1,759 & 62 & -22 & 6 & 15.80 & $<0.05^{*}$ & 2.95 \\
& 2 & Right STG & & 68 & -14 & -4 & 12.30 & $<0.05^{*}$ & 1.84 \\
& 3 & Right STG & & 64 & -6 & -8 & 10.26 & $<0.05^{*}$ & 1.16 \\
\hline
\end{tabular}

*Significant at set, cluster and voxel level (Friston et al. 1996).

Listening to babble noise or ' $\mathrm{N}$ ' task resulted in widespread activation in the middle temporal gyrus (MTG) and superior temporal gyrus (STG) in the left and right temporal lobes. The activation appears to be spatially symmetrical but the number of activated voxels $\left(K_{\mathrm{E}}\right)$ is higher in the right temporal lobe as compared to the left temporal lobe.The height extent of activation observed at the coordinates of maximum intensity in each cluster, however, was determined to be higher in the left temporal lobe (Table 1). This characteristic of activation in the left temporal lobe is accentuated in the $t$-statistics for the three maxima and the more intense activation pattern as shown in Table 1 and Fig. 3 respectively. The stimulus efficacy $(\varepsilon)$ was also determined to be higher for the 
maximum intensity voxel in the left hemisphere (Table 1) as compared to the maximum intensity voxel in the right hemisphere.

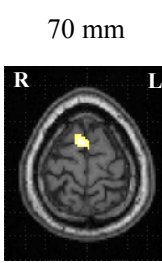

$20 \mathrm{~mm}$
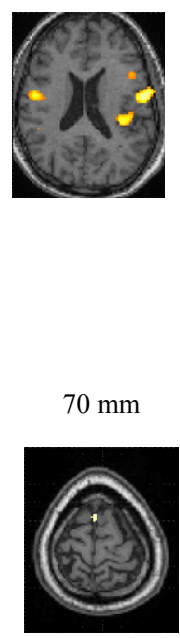

$20 \mathrm{~mm}$

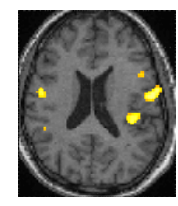

$60 \mathrm{~mm}$

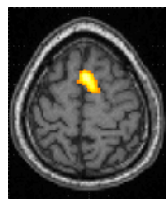

$10 \mathrm{~mm}$

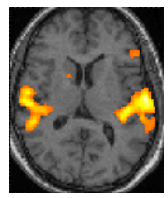

$60 \mathrm{~mm}$

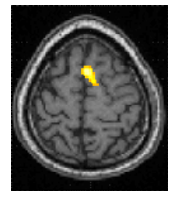

$10 \mathrm{~mm}$

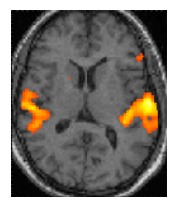

$50 \mathrm{~mm}$

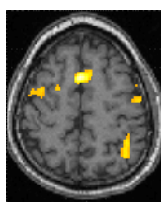

$0 \mathrm{~mm}$

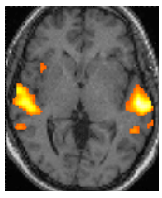

(a)

$50 \mathrm{~mm}$

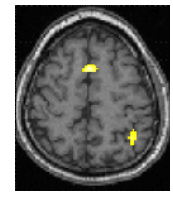

$0 \mathrm{~mm}$

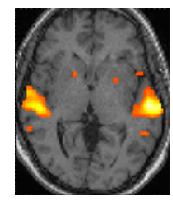

$40 \mathrm{~mm}$

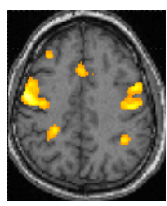

$-10 \mathrm{~mm}$

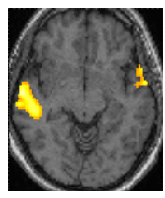

$40 \mathrm{~mm}$

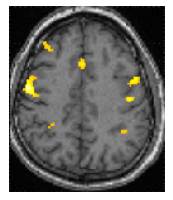

$-10 \mathrm{~mm}$

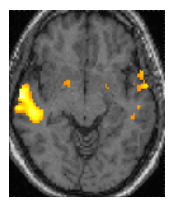

$30 \mathrm{~mm}$

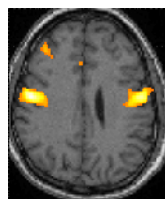

$-20 \mathrm{~mm}$

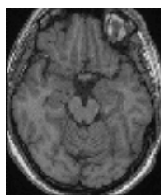

$30 \mathrm{~mm}$

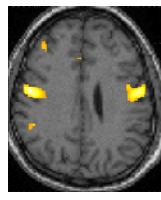

$-20 \mathrm{~mm}$

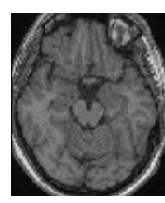

(b)

Figure 4. Activated brain regions when the subject performs (a) working memory task in quiet (WMQ) and (b) in noise (WMN); ( $\mathrm{R}=$ Right, $\mathrm{L}=\mathrm{Left})$. The statistical images (colored) were overlaid decendingly onto the paticipant's axial $(z=70$ to $z=-20 \mathrm{~mm})$ normalised T1-weighted images. The scale for the $t$-value is similar as in Fig. 3.

Figs. 4(a) and (b) show, respectively, the brain activations that have been captured during the execution of working memory in quiet (WMQ) and in the presence of babble noise (WMN). The images are presented neurologically from which one should note that the left-hand side of the images represent the left side of the brain. The images were obtained after thresholding the height extent of activation at $t=5.21\left(p_{\mathrm{FWE}}<0.05\right.$ for each voxel, corrected for multiple comparisons). The spatial extent threshold was not used on these images to avoid losing important clusters of voxels that might be involved in, or associated with, execution of the working memory task. By comparing Figs. 4(a) and (b), it can be said that WMQ and WMN has resulted in similar activated clusters of voxels or regions in the brain. The spatial extent of activation for the clusters is about the same from the 20-mm level down to $-20-\mathrm{mm}$ level. However, the activation from the $20-\mathrm{mm}$ level to $70-\mathrm{mm}$ level is relatively wider (containing more activated voxels) during WMQ as compared to WMN. A detailed analysis result for WMQ and WMN is given in Table 2 and described in the following paragraphs. 
Table 2. Characteristics of brain activation for (a) WMQ and (b) WMN as shown in Fig. 4. BA= Brodmann Area; TE1.1 = primary auditory area; hIP1 = inferior parietal sulcus; IPC $=$ Inferior parietal cortex; IFG $=$ Inferior frontal gyrus.

Table 2 (a).

\begin{tabular}{|c|c|c|c|c|c|c|c|c|c|}
\hline Cluster & $\begin{array}{c}\text { Local } \\
\text { maximum }\end{array}$ & Region & $K_{\mathrm{E}}$ & $x$ & $y$ & $z$ & $t$-value & $p_{\text {FWE-corr. }}$ & $\varepsilon / s^{-1}$ \\
\hline \multirow[t]{3}{*}{1} & 1 & Left MTG & 3,521 & -56 & -20 & 4 & 14.31 & $<0.05^{*}$ & 3.33 \\
\hline & 2 & Left BA3a & & -54 & -10 & 24 & 13.33 & $<0.05^{*}$ & 2.06 \\
\hline & 3 & Left TE1.1 & & -36 & -36 & 16 & 11.51 & $<0.05^{*}$ & 1.67 \\
\hline \multirow[t]{3}{*}{2} & 1 & Right BA3b & 3,423 & 56 & -10 & 32 & 12.12 & $<0.05^{*}$ & 2.48 \\
\hline & 2 & Right STG & & 58 & -22 & 4 & 11.46 & $<0.05^{*}$ & 3.59 \\
\hline & 3 & Right STG & & 64 & -10 & -4 & 11.35 & $<0.05^{*}$ & 1.61 \\
\hline \multirow[t]{3}{*}{3} & 1 & Right SMA & 1,025 & 2 & 8 & 58 & 11.86 & $<0.05^{*}$ & 2.49 \\
\hline & 2 & Right BA6 & & 6 & 12 & 52 & 9.85 & $<0.05^{*}$ & 1.84 \\
\hline & 3 & Left BA6 & & -4 & 0 & 62 & 9.11 & $<0.05^{*}$ & 2.00 \\
\hline 4 & 1 & Right MTG & 176 & 40 & 34 & 34 & 7.80 & $<0.05^{*}$ & 2.14 \\
\hline \multirow[t]{3}{*}{5} & 1 & hIP1 & 226 & -38 & -50 & 44 & 7.69 & $<0.05^{*}$ & 1.44 \\
\hline & 2 & IPC (PFm) & & -38 & -62 & 48 & 6.52 & $<0.05^{*}$ & 1.50 \\
\hline & 3 & $\mathrm{BA} 2$ & & -42 & -42 & 48 & 6.01 & $<0.05^{*}$ & 0.87 \\
\hline 6 & 1 & - & 111 & 32 & -42 & 40 & 7.30 & $<0.05^{*}$ & 1.02 \\
\hline 7 & 1 & Left IFG & 73 & -42 & 14 & 22 & 6.93 & $<0.05^{*}$ & 0.69 \\
\hline 8 & 1 & Right Putamen & 37 & 18 & 8 & 6 & 6.91 & $<0.05^{*}$ & 0.90 \\
\hline 9 & 1 & Left MTG & 69 & -52 & -50 & 0 & 6.76 & $<0.05^{*}$ & 0.80 \\
\hline 10 & 1 & Left IFG & 52 & -46 & 28 & 8 & 6.13 & $<0.05^{*}$ & 0.68 \\
\hline 11 & 1 & - & 21 & 28 & 2 & 48 & 6.08 & $<0.05^{*}$ & 0.77 \\
\hline
\end{tabular}

*Significant at set, cluster and voxel level (Friston et al. 1996).

Table 2 (b).

\begin{tabular}{cclccccccc}
\hline Cluster & $\begin{array}{c}\text { Local } \\
\text { maximum }\end{array}$ & Region & $K_{\mathrm{E}}$ & $x$ & $y$ & $z$ & $t$-value & $p_{\text {FWE-corr. }}$ & $\varepsilon / \mathrm{s}^{-1}$ \\
\hline 1 & 1 & Left STG & 2,868 & -58 & -18 & 6 & 16.49 & $<0.05^{*}$ & 5.33 \\
& 2 & Left BA3a & & -54 & -10 & 26 & 10.95 & $<0.05^{*}$ & 3.58 \\
& 3 & Left TE1.1 & & -36 & -36 & 14 & 10.88 & $<0.05^{*}$ & 3.13 \\
2 & 1 & Right STG & 2,510 & 54 & -24 & 2 & 12.40 & $<0.05^{*}$ & 3.48 \\
& 2 & Right STG & & 64 & -10 & -4 & 11.58 & $<0.05^{*}$ & 3.70 \\
& 3 & Right BA6 & & 58 & -6 & 36 & 9.78 & $<0.05^{*}$ & 2.58 \\
3 & 1 & Right SMA & 516 & 2 & 8 & 58 & 9.87 & $<0.05^{*}$ & 2.31 \\
& 2 & Left BA6 & & -4 & 0 & 62 & 6.91 & $<0.05^{*}$ & 2.00 \\
& 3 & Right SMA & & 14 & 8 & 76 & 6.89 & $<0.05^{*}$ & 1.75 \\
4 & 1 & Right MTG & 135 & 40 & 34 & 36 & 7.26 & $<0.05^{*}$ & 2.51 \\
5 & 1 & IPC (PFm) & 64 & 52 & -44 & 26 & 6.97 & $<0.05^{*}$ & 1.11 \\
6 & 1 & Right Putamen & 92 & 18 & 10 & 4 & 6.97 & $<0.05^{*}$ & 0.78 \\
& 2 & - & & 16 & 0 & -6 & 5.92 & $<0.05^{*}$ & 0.73 \\
& 3 & Right & & 22 & 2 & -12 & 5.61 & $<0.05^{*}$ & 1.16 \\
7 & & Amygdala & & & & & & & \\
8 & 1 & Right IFG & 36 & -44 & 12 & 22 & 6.61 & $<0.05^{*}$ & 1.27 \\
9 & 1 & hIP1 & 132 & -40 & -52 & 46 & 6.54 & $<0.05^{*}$ & 2.03 \\
10 & 1 & Left MTG & 33 & -52 & -50 & 0 & 6.22 & $<0.05^{*}$ & 1.43 \\
11 & 1 & BA45 & 27 & -50 & 28 & 8 & 6.16 & $<0.05^{*}$ & 1.17 \\
\hline
\end{tabular}

*Significant at set, cluster and voxel level (Friston et al. 1996).

There are altogether 18 activated clusters of voxels for WMQ and 15 for WMN that survived the height threshold of $t=5.21$ ( $p_{\mathrm{FWE}}<0.05$ for each voxel, corrected for multiple comparisons) from which the respective anatomical regions for 11 highly activated clusters are shown in Table 2 (a) and (b). By comparing and contrasting WMQ and WMN, the first four clusters of voxels with the highest $t$-values appear to be similar. However, a marked increase in the number of activated voxels can be seen for WMQ in contrast to WMN. The coordinates of the maxima of first two clusters for both WMQ and WMN are quite similar to one another. These two clusters exhibited widespread activation in the temporal and frontal regions. In addition, a relatively intense activation is also observed in the supplementary motor area (SMA) and the number of activated voxels for WMQ in this region is almost twice the number of activated voxels for WMN. The rest of the brain areas that 
are activated with lower $t$-value and less number of activated voxels are shown in Table 2 .

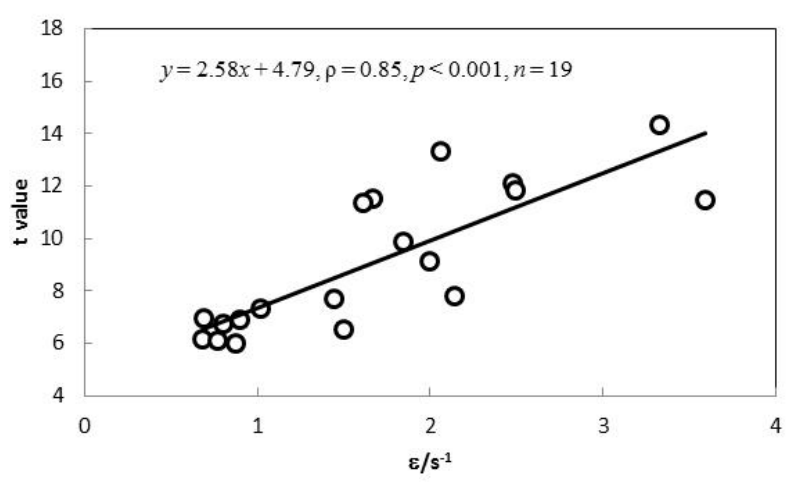

(a)

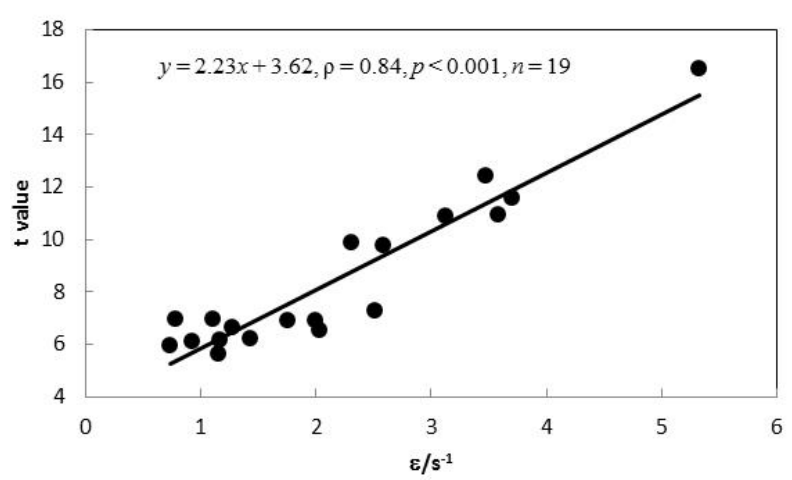

(b)

Figure 5. Linear correlation between the $t$ value and the mean stimulus efficacy for a) WMQ and b) WMN conditions

Figure 5(a) and (b) illustrate the relationship between the effect size ( $t$ value) and the mean stimulus efficacy ( $\varepsilon$ ) calculated for the respective areas as tabulated in Table 2 for both WMQ and WMN conditions. There exists a linear, significant $(p<0.001)$, positive relationship between the $t$ value and the mean stimulus efficacy for both WMQ and WMN conditions with a steeper slope $(m)$ for WMQ $(m=2.58 \mathrm{~s})$ as compared to WMN $(m=2.23 \mathrm{~s})$. The Spearman's correlation coefficient for WMQ $(\rho=0.85)$ is, however, not different from that of WMN $(\rho=$ $0.84)$. 

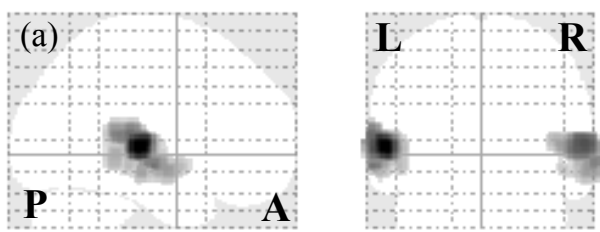

$-58 /-22 / 6$

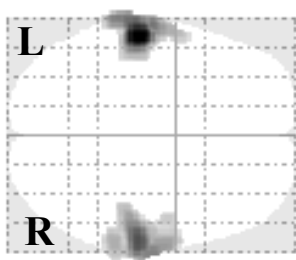

$\operatorname{SPM}\left\{T_{79}\right\}$
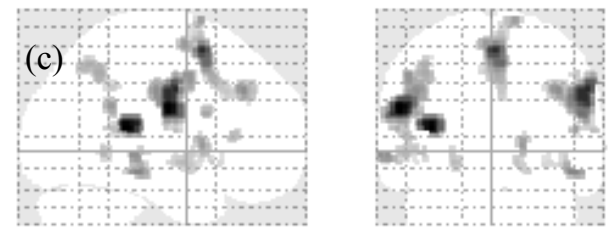

$-54 /-10 / 26$

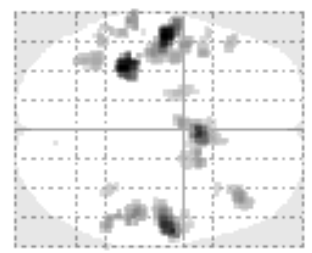

\section{$\operatorname{SPM}\left\{T_{79}\right\}$}
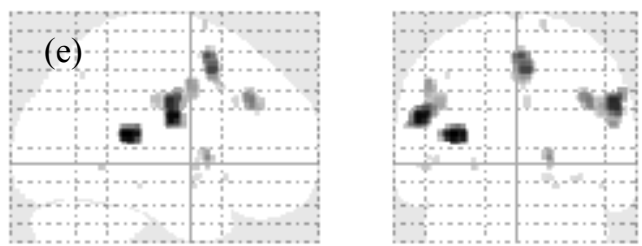

$-34 /-34 / 16$

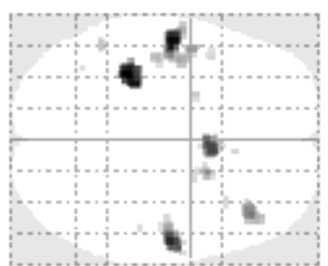

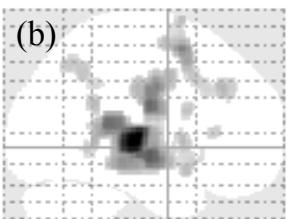

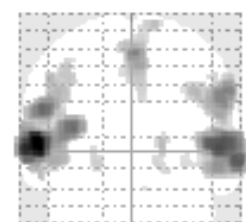

$-58 /-18 / 6$

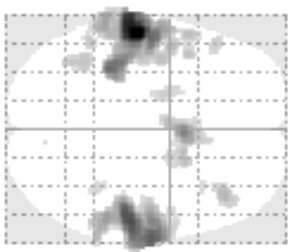

$\operatorname{SPM}\left\{\mathrm{T}_{79}\right\}$
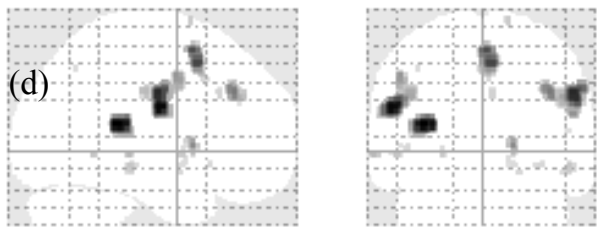

$-34 /-34 / 16$

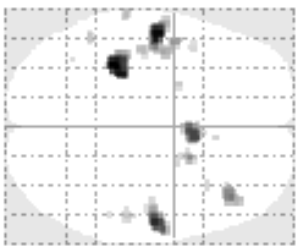

$\operatorname{SPM}\left\{\mathrm{T}_{79}\right\}$
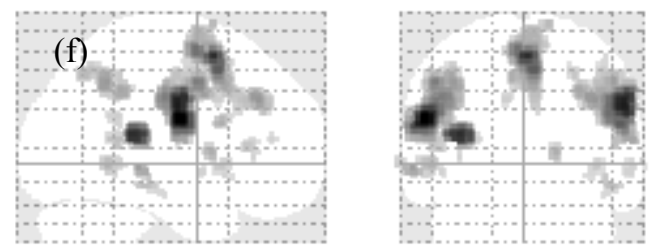

$-34 /-34 / 16$

$\operatorname{SPM}\left\{T_{79}^{2\{\mathrm{Ha}: \mathrm{k}=2\}}\right\}$

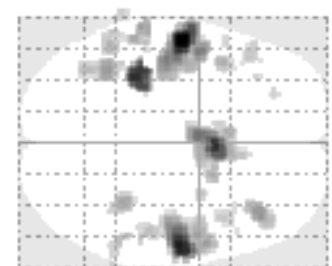

Figure 6. The MIP of the brain activation for the effects of (a) N, (b) WMN, (c) WMN - N, (d) WMN $>$ N, (e) conjunction of $\mathrm{c}$ and $\mathrm{d}$ and (f) WMQ - N. $(\mathrm{P}=$ Posterior, $\mathrm{A}=$ Anterior, $\mathrm{L}=$ Left, $\mathrm{R}=$ Right $)$

Another way of visualising brain activation is by using maximum intensity projection (MIP) or glass images. Figs. 6( $\mathrm{a}$ and $\mathrm{b}$ ) show the MIPs for $\mathrm{N}$ and WMN conditions. Note that while still being in the same region, the coordinates of maximum intensity have shifted from $-58 /-22 / 6$ to $-58 /-18 / 6$. To obtain the effects due to working memory; thus two MIPs were used to subtract Fig. 6(a) from Fig. 6(b) (WMN minus N). The results are shown in Fig. 6(c). Comparing Figs. 6(b) and 6(c), one notes that the effects of babble noise have been subtracted from Fig. 6(b) and the coordinates of maximum intensity, previously at $-58 /-22 / 6$ have shifted to $-54 /-10 / 26$. What is 
left in Fig. 6(c) is assumed to be the true effects of the working memory task in noise (to be compared with Fig. 6(b)). Fig. 6(c) can be compared with Fig. 6(d) which shows the results of the contrast WMN $>$ N. The contrast $\mathrm{WMN}>\mathrm{N}$ is used to search for voxels that are more active in the WMN condition than in the $\mathrm{N}$ condition at a certain significance level. It can be clearly seen that Fig. 6(d) is comparable to Fig. 6(c) in terms of the most prominent clusters but with less background noise in the activation. One can see that the coordinates of maximum intensity between Figs. 6(c) and (d) were also different.

Fig. 6(e) is the conjunction of the effects of Figs. 6 (c) and (d). Conjunction analysis was performed to confirm the activation areas that solely belong to the true effects of working memory in noise. No significant difference was found between Fig. 6(d) and (e). Fig. 6(e) therefore represents the spatial extent of activation (or the true effects) in the brain when the participant performs the working memory task in the noisy (babble noise) condition. Fig. 6(f) depicts results of the working memory in quiet (WMQ) minus N. Comparing Fig. 6(e) and Fig. 6(f) and contrasting Fig. 6(c) and Fig. 6(f) it appears that all areas involved in the working memory task in noise are also involved in the same task in quiet; moreover, the working memory task in quiet activates a wider extent of brain areas compared to the working memory performed in a noisy background.

(a)
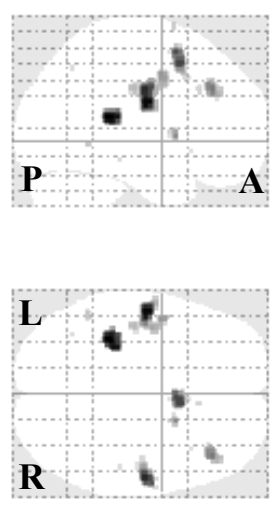

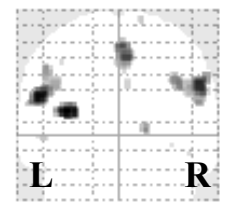

$\mathbf{L}$ $\mathbf{R}$ $\operatorname{SPM}\left\{T_{79}^{3\{\mathrm{Ha}: \mathrm{k}=3\}}\right\}$ (b)

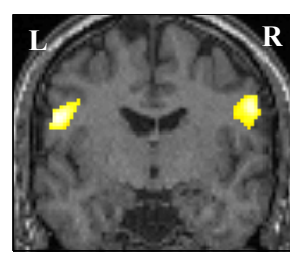

TE1.1

$y=10 \mathrm{~mm}$

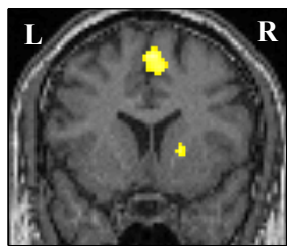

BA6/SMA (top) and

putamen (bottom)

$y=-10 \mathrm{~mm}$

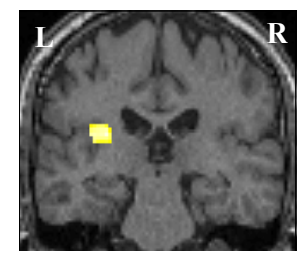

BA3a (left) and BA4p (right)

$y=34 \mathrm{~mm}$

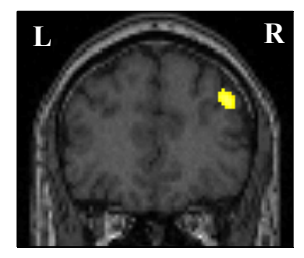

Superior frontal gyrus

Figure 7. The MIP for the conjunction of the effects of WMQ, WMN and $M N>N$ minus $N$ and (b) Participant's coronal T1-weighted images (a) at four $y$ positions showing the region associated with working memory. The scale for the $t$-value is similar as in Fig. 3 ( $\mathrm{P}=$ Posterior, $\mathrm{A}=$ Anterior, $\mathrm{L}=\mathrm{Left}, \mathrm{R}=$ Right)

Fig. 7(a) illustrates the true effects of the working memory task, regardless of whether the task is performed in quiet or noisy (babble noise) conditions. The MIPs are the results obtained from the conjunction of the effects of WMQ, WMN and WMN $>$ N, minus the effects of N. Fig. 7(b) is Fig. 7(a) overlaid onto the participant's coronal slices. The four slices at different $y$ locations clearly indicate the role of TE 1.1, BA3a, BA4p, SMA, putamen and SFG when the participant was performing the working memory task. It is evident from Fig. 7(a) that TE1.1 $(-34 /-34 / 16)$ is the cluster that shows the highest intensity as compared to the others, indicating the important role of this area as a processing centre in the execution of the working memory task used in this study.

\subsection{Psychophysiologic Interaction (PPI)}

The activated areas that have been obtained from conjunction analysis of the effects of the working memory task in the left and right hemispheres are the left and right superior temporal gyrus (STG) as the source region, the left and right TE1.1, left BA3a, right BA4p, right superior frontal gyrus (SFG), right putamen and SMA. Due to the midsagittal activation for the SMA, it is considered that the same SMA is involved in the connectivity in the left and right hemispheres, during both WMQ and WMN. The goal is to use PPI to examine the change in 
effective connectivity between the STG as the input region and all the other activated regions as the target regions in the left and right hemisphere while the participant is performing the working memory task under the experimental treatment of less attention (WMQ) and more attention (WMN).

Table 3. Statistics obtained from regression analyis on PPI data between the target region and the region of interest

\begin{tabular}{|c|c|c|c|c|c|}
\hline & $b$ & $\beta$ & $r^{2}$ & $p$ & $95 \% \mathrm{CI}$ \\
\hline \multicolumn{6}{|c|}{ Left hemiphere areas in quiet } \\
\hline Left TE1.1 vs. left STG & 0.504 & 0.632 & 0.400 & $<0.001$ & $0.391-0.616$ \\
\hline Left BA3a vs. left STG & 0.491 & 0.637 & 0.405 & $<0.001$ & $0.383-0.600$ \\
\hline SMA vs. left STG & 0.479 & 0.568 & 0.322 & $<0.001$ & $0.352-0.605$ \\
\hline \multicolumn{6}{|c|}{ Left hemisphere areas in noise } \\
\hline Left TE1.1 vs. left STG & 0.552 & 0.661 & 0.437 & $<0.001$ & $0.438-0.667$ \\
\hline Left BA3a vs. left STG & 0.519 & 0.653 & 0.427 & $<0.001$ & $0.409-0.629$ \\
\hline SMA vs. left STG & 0.506 & 0.591 & 0.349 & $<0.001$ & $0.380-0.631$ \\
\hline \multicolumn{6}{|c|}{ Right hemisphere areas in quiet } \\
\hline Right BA4p vs. right STG & 0.429 & 0.568 & 0.322 & $<0.001$ & $0.316-0.543$ \\
\hline Left SFG vs. right STG & 0.588 & 0.541 & 0.292 & $<0.001$ & $0.421-0.755$ \\
\hline SMA vs. right STG & 0.471 & 0.600 & 0.361 & $<0.001$ & $0.357-0.586$ \\
\hline Right putamen vs. right STG & 0.202 & 0.535 & 0.286 & $<0.001$ & $0.144-0.260$ \\
\hline \multicolumn{6}{|c|}{ Right hemisphere areas in noise } \\
\hline Right BA4p vs. right STG & 0.424 & 0.574 & 0.330 & $<0.001$ & $0.314-0.534$ \\
\hline Left SFG vs. right STG & 0.532 & 0.500 & 0.250 & $<0.001$ & $0.364-0.700$ \\
\hline SMA vs. right STG & 0.437 & 0.572 & 0.328 & $<0.001$ & $0.323-0.551$ \\
\hline Right putamen vs. right STG & 0.163 & 0.426 & 0.181 & $<0.001$ & $0.100-0.226$ \\
\hline
\end{tabular}

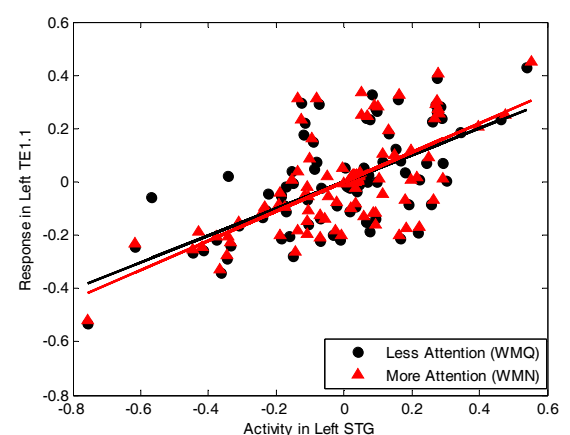

(a)

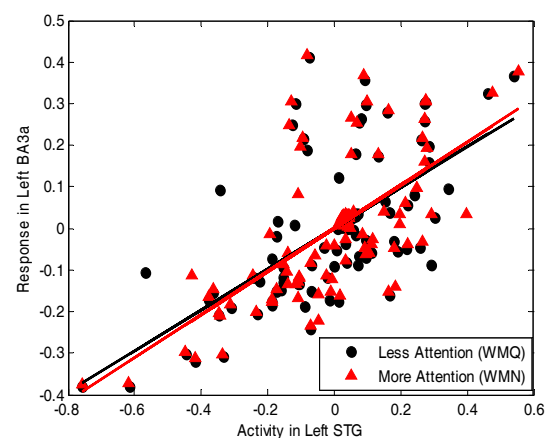

(b)

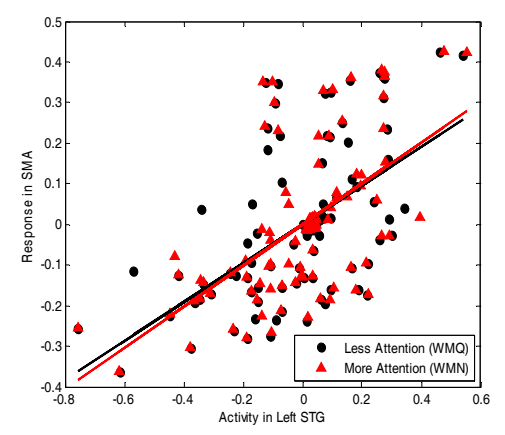

(c)

Figure 8. Psychophysiologic interaction between left STG and (a) TE1.1, (b) BA3a and (c) SMA without (dark) and with (red) the presence of babble noise 

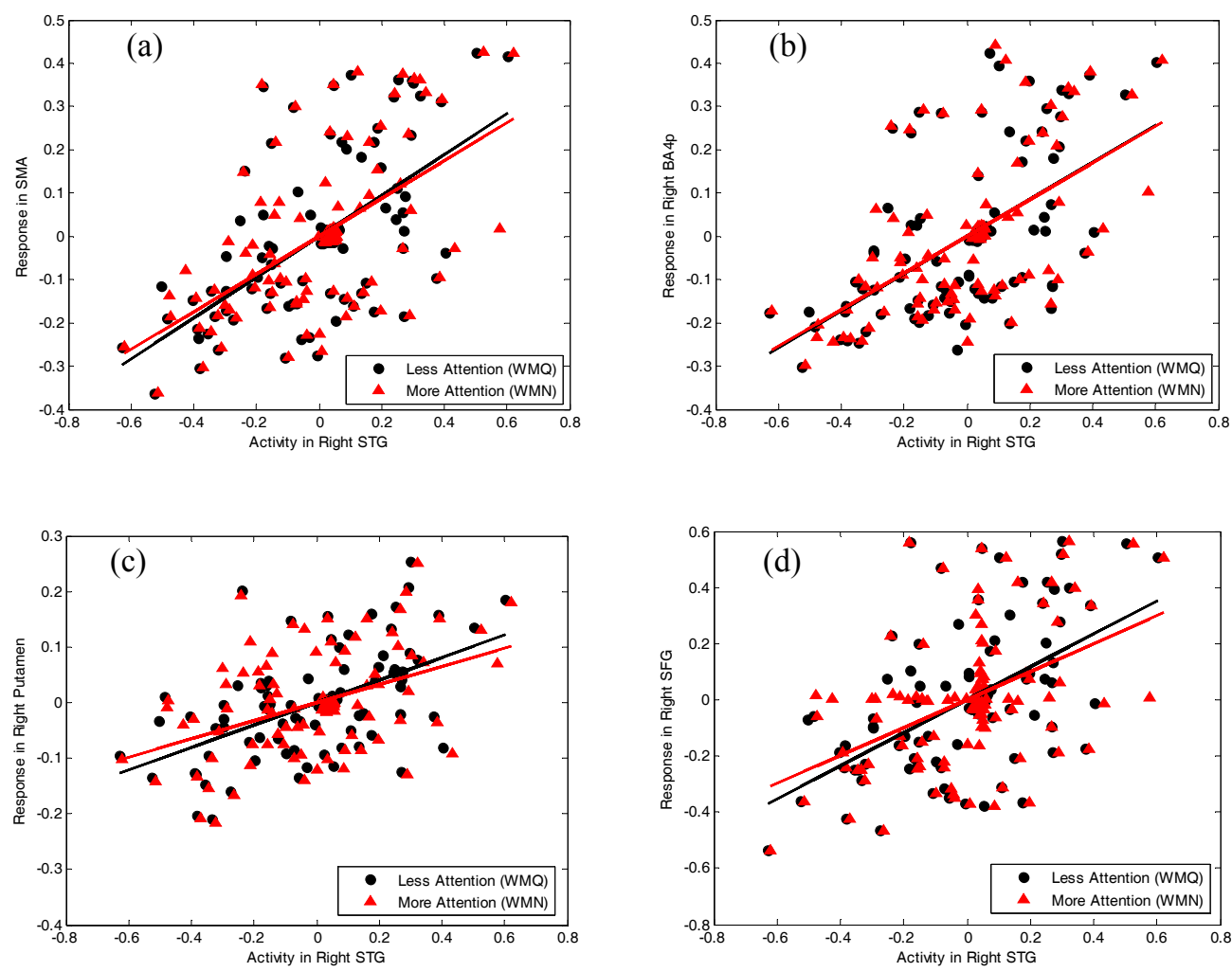

Figure 9. Psychophysiologic interaction between right STG and (a) right SMA, (b) BA4p, (c) right putamen and (d) right SFG without (dark) and with (red) the presence of babble noise

Figs. 8 illustrate the response in the left TE1.1, BA3a and SMA plotted as a function of the activity in the left STG obtained from psychophysiologic interaction (PPI) analyses. It can be said that the increase in activity in the left STG is associated with increases in the responses in TE1.1, BA3a and SMA both in noise and in quiet. The PPI during execution of the working memory task seemed to be increased in noise (more attention) as compared to in quiet (less attention) as shown by the steeper slope of the red lines. Similar relationships are obtained in the right hemisphere between the right STG and BA4p, SMA, SFG and putamen as shown in Fig. 8. However, these right hemisphere regions indicated an increase in the slope during less attention (dark lines) as compared to more attention except for the BA4p - STG relationship in which the two lines have almost equal slopes.

The statistics characterising all of the slopes shown in Fig. 8 and 9 are tabulated in Table 3 (a) - (d) as obtained from simple linear regression analysis. All the data fulfill the assumptions of normality, linearity and homoscedasticity for the predicted values and residuals. It can be said that the results obtained from simple linear regression analysis have shown that there is a significant, positive and linear relationship between the responses in all the target regions and the activity in the STG $(n=120, p<0.001,95 \% \mathrm{CI})$. However, the proportion of the variation in the response of the target regions that can be explained by the activity in the STG, according to the linear regression model is less than $50 \%(18-44 \%)$ due to a large dispersion of the data.

\section{Discussion}

The MNI $x, y, z$ coordinates shown in Table 1 have been found to be located in the auditory associated areas, mainly in the middle and superior temporal gyri of the left and right temporal lobes, according to Specht \& Reul (2003) in which brain activations due to attentive listening to tones, sounds and words were comprehensively recorded. The two maxima in the left and right temporal lobes that mirrored each other show that the processing centres for attentive listening to babble noise are the same in both hemispheres, at least for this particular participant. The auditory cortex can be functionally and structurally divided into three main parts which are the primary, secondary and tertiary auditory cortices, forming concentrically around one another. The most inner part is the primary auditory cortex and the most outer part is the tertiary auditory cortex. Widespread activation 
observable in the bilateral temporal lobes (Fig. 2) can be explained as due to the commonly known tonotopic characteristic of the auditory cortex especially in the primary auditory cortex. Tonotopic characteristic means that certain cells in the auditory cortex are sensitive to specific frequencies. The babble noise spectrum used in this study consists of a wide range of frequencies which in turn excite many cell regions that are responsible for each particular frequency, resulting in widespread activation. The tonotopic characteristic for the auditory cortex was evident in Langers et al. (2007) and Langers \& van Dijk (2012). In their study, a prominent low-to-high frequency tonotopic gradient was observed in the lateral to medial direction of the transverse temporal gyrus (TTG), in the primary auditory field and planum temporale. It has been demonstrated (Specht \& Reul 2003) that the primary auditory cortex responded to a broad range of auditory stimuli while the secondary auditory cortex responded to stimuli with complex spectral dynamics. Similar temporal lobe activations (average) have also been found in our previous study using a white noise stimulus on five right handed male participants (Yusoff et al. 2011). The absence of brain activations in the frontal and parietal lobe regions indicate that listening to babble noise does not involve any higher cognitive function over and above baseline comparisons. The activation in the auditory cortex may also indicate that the participant's auditory processing is working well in conjunction with the participant's good hearing condition which has been confirmed by the auditory test performed on the participant's left and right ears.

Interestingly, a higher number of activated voxels in the right auditory cortex is accompanied by a lower $t$ statistic at the point (voxel) of maximum intensity as compared to the left auditory cortex which has fewer activated voxels but with higher $t$ statistic at the point of maximum intensity (Table 1). Not only did the number of activated voxels and the $t$ statistic for the two hemispheres differ in values, but the significance of these effects was very large. Based on previous findings (Hwang et al. 2005), more voxels have been found to be activated in the right temporal lobe as compared to the left temporal lobe when noise is used as stimulus, due its non-speech property (Hwang et al. 2005). Speech stimulus, on the other hand, will most likely activate the left temporal lobe relatively higher than the right temporal lobe especially when perception of words comes into play and only if the stimulus task is linguistically demanding (Hwang et al. 2005). The $t$ value for the voxel of maximum intensity is at odds with the observed number of activated voxels in both temporal lobes but in good agreement with the mean stimulus efficacy $(\varepsilon)$ value. The mean stimulus efficacy is defined as the potency of the stimulus in eliciting a neuronal response (Friston et al. 2000). It reflects the ability of the ensuing synaptic activity to induce a signal. It seems that the left hemisphere temporal lobe has a stronger ability in the processing of babble noise as compared to the right temporal lobe. One possible explanation for the increase in the mean stimulus efficacy is that the babble noise used in this study was still in the range intelligible to the participant who was young and healthy and was able to be processed by the left hemisphere temporal lobe. It is perhaps this mean stimulus efficacy of the left hemisphere temporal lobe that causes the signal intensity to be higher in the left temporal lobe, hence the higher $t$ value. The fact that signal intensity increases correspondingly with the mean stimulus efficacy is evident in Fig. 5. The underlying mechanism will be discussed in the following paragraph.

While maintaining the activation in the cortical auditory regions, performing a phonological working memory task in quiet (WMQ) and noisy (WMN) conditions has evoked responses in many other cortical regions as tabulated in Table 2 (a) and (b) and can be clearly seen in Figs. 4(a) and (b). Among others are TE1.1 which is known as the primary auditory area, supplementary motor area (SMA), Brodmann Area (BA) 3a and 3p, inferior parietal cortex (IPC) and putamen. These regions have been found to be activated during working memory tasks in several previous studies (Gaab et al. 2003; Karlsgodt et al. 2005; Toepper et al. 2010). Nevertheless, the differences in the brain activated regions during WMQ and WMN can hardly be seen. This could be due to the same regions in the brain responding to the phonological working memory task used in this study whether performed in quiet or in noise. The result is indirectly supported by the behavioural results which indicate similar participant's performance during WMQ and WMN. The spatial extent of activation that seems to be wider from the 70-mm level down to 20-mm level during WMQ, involves the first four activation clusters shown in Table 2 (a). A larger activation area means that a larger number of brain cells are recruited in performing the task in quiet as compared to in noise. In a finger tapping task (Jancke et al. 1998), it was found that a larger brain area was involved in performing the tapping at $3 \mathrm{~Hz}$ as compared to at $1 \mathrm{~Hz}$. They concluded that faster movement involves the recruitment of more motor units and will therefore activate a greater number of voxels. Their findings were later reconfirmed (Lutz et al. 2005). In this study, a higher number of activated voxels reflects the higher sensitivity of the brain areas in perceiving the stimulus and later performing the task in quiet. During WMN, it can be said that the participant used a lesser volume of the respective brain regions in accomplishing the working memory task due to the distraction from the noise. Brain areas that are supposed to be involved in processing the phonological working memory task used in this study are less sensitive when the stimulus is 
embedded in noise. In other words their ability to perceive what is delivered is relatively lower in noise than in quiet. The stimulus-to-noise ratio (SNR) used in this study was $5 \mathrm{~dB}$. Furthermore, a lower noise intensity $\left(\mathrm{I}_{\mathrm{N}}\right)$ as compared to stimulus intensity $\left(\mathrm{I}_{\mathrm{S}}\right)$ used in this study did not enhance the spatial extent of activation as found in previous studies on multiple participants (Wong et al. 2009; Wong et al. 2008). Enhancement of spatial activation occurs in certain brain regions when the participants performed working memory (Abd Hamid et al. 2011) or speech perception (Wong et al. 2009; Wong et al. 2008) tasks in noisy backgrounds ( $\mathrm{SNR}=+3 \mathrm{~dB}$ and $-5 \mathrm{~dB}$ respectively) due to an increase in participants' effort in attending and performing the task while being distracted by noise. One possible explanation for the effects observed for this particular participant is that the sensitivity of the particular brain region in perceiving and performing the phonological working memory task in quiet has exceeded the activation enhancement in performing the task in noisy background. The $+5 \mathrm{~dB}$ SNR used in the present study may not be distracting enough for the participant who is relatively young and has good hearing.

This argument is indirectly supported by the steeper slope $(m)$ of the $t$ value vs. $\varepsilon$ plot for WMQ, $m=2.58 \mathrm{~s}$, as compared to the less steeper slope of the $t$ value vs. $\varepsilon$ plot for WMN, $m=2.23 \mathrm{~s}$, as shown by Figs. 5(a) and (b). A steeper slope for WMQ means that the blood oxygenation level dependent (BOLD) signal intensities, hence the hemodynamic responses, across the activated regions change relatively larger during WMQ as compared to during WMN due to a better efficiency of the ensuing synaptic activity to induce the signal in quiet. The mean stimulus efficacy thus represents the increase in perfusion signal elicited by neuronal activity, expressed in terms of number of evoked transients per second (Friston et al. 2007). From the plots, it appears that the extent of signal change across activated regions is larger in quiet as compared to in noise for the same change in the mean stimulus efficacy. However, the distributions of data for the two conditions are about the same, resulting in similar values of the Spearman coefficient for both plots.

The participant-specific brain activation characteristic discussed above may differ from participant to participant. However, in view of the brain activation obtained from WMQ and WMN conditions that have been averaged over 20 measurements, the brain activation results can be said to be valid, at least for this particular participant due to the high sensitivity of sparse imaging technique used in this study in capturing the change in the magnetic resonance signal intensity during the task performance (Gaab et al. 2007; Mueller et al. 2011).

Further analyses conducted using contrast comparison and conjunction analysis on our participant revealed two additionally important findings reflecting 1) the true effects of phonological working memory task performance in babble noise and in quiet and 2) that the brain areas involved in performing the working memory task are similar regardless of whether the task was performed in quiet or in noise. The six brain areas found to be significantly activated at corrected $\alpha=0.05$ are left TE1.1, left BA3a, right BA4p, BA6/SMA, putamen and SFG. These six brain areas appear to be reproducible using WMN - N, WMN $>\mathrm{N}$ and WMQ - N contrasts, using conjunction of the effects of WMN - N and WMN $>\mathrm{N}$ and the conjunction of the effects of WMQ, WMN and $\mathrm{WMN}>\mathrm{N}$, minus the effects of $\mathrm{N}$. The difference between WMN $-\mathrm{N}$ and $\mathrm{WMN}>\mathrm{N}$ contrasts is that the former is looking for voxels or brain areas that are activated during WMN after subtracting it from areas that are activated during $\mathrm{N}$, while the latter is looking for voxels that are more (significantly) activated in WMN than in $\mathrm{N}$. What is revealed by taking the conjunction between these two contrasts should be the areas that are involved in processing the phonological working memory task stimulus delivered to the participant during the scan.

The conjunction of the effects of WMQ, WMN and WMN $>\mathrm{N}$, minus the effects of $\mathrm{N}$ confirmed the earlier results and is reconfirmed by the WMQ - $\mathrm{N}$ contrast. Conjunction analysis permits the determination of the areas in the brain for a specific function that are common in all tasks under study. It allows for the identification of areas that share a specific function in a participant. For the WMQ - N contrast, even though the effects of $\mathrm{N}$ are not inherited in WMQ, the activation obtained can be compared with WMN - N and WMN $>\mathrm{N}$ contrasts because what is taken out from the WMQ - N contrast are voxels that are involved in listening or, in particular, in babble noise, leaving behind voxels that do not belong to the listening task when performed during $\mathrm{N}$ condition.

The exact location of the six brain areas found to be involved in the phonological working memory task can be observed in Fig. 7 from which the statistical images are overlaid coronally onto the participant's normalised brain images. Out of the six areas, the left TE1.1, left BA3a, BA6/SMA and right BA4p have shown an increase in the number of activated voxels when the task is performed in quiet as compared to in noise, see Table 2. In relation to the above discussion, it can be said that the sensitivity of these particular brain regions in perceiving and performing the phonological working memory task in quiet increases, which is reflected by an increase in the total volume of the activation area.

As shown in Fig. 2, the psychophysiologic interaction (PPI) explains the regionally specific neuronal responses in the target regions in terms of the interaction between influences from a source region and task-related 
parameters such as babble noise. In this study, the regionally specific neuronal responses are measured in the brain regions shown in Fig. 7 which are the left TE1.1, left BA3a, SMA, right BA4p, right SFG and right putamen, also known as the target regions. The response in these regions is said to be influenced by the interaction between the activity in the STG, the source region, and the task-related parameter which is the babble noise. The left and right STG are chosen to be the source region whose activity interacts with babble noise as well as with the working memory stimulus due to the source region's well known functional role in processing verbal and nonverbal stimuli. Furthermore, STG is also an area in which the primary and secondary auditory areas are located and from where the input (or stimuli) traverses to the other areas in the brain. The activity in STG, therefore on the one hand interacts with babble noise to evoke responses in the target areas in which babble noise is said to modulate the interaction between the STG with other regions connected to it. On the other hand, babble noise also has a direct influence on the target areas as shown in Fig. 2. Thus, it can be said that the responses in the target regions reflect the combined effect of the interaction between the source regions and babble noise and the influence of babble noise itself on target regions.

To measure the influence of the activity in the source region (in this study the left and right STG) on the response of the target regions (as shown in Fig. 7), the PPI data of the target region (as the dependent variable) is plotted against the PPI of the source region (as the independent variable). Figs. $8(a-c)$ and $9(a-d)$ illustrate psychophysiologic interaction (PPI) between the two distal brain areas, namely each of the areas in Fig. 7 vs. the left or right STG. A linear increase in the response in all the target regions with the increase in activity in the source region as depicted provides evidence in support of a direct influence of the activity of the source region on the response of the target regions. Linearity is shown in all the plots regardless of whether the task is performed in quiet (dark lines) or in noise (red lines). The slope $(b)$ of these plots reflects the influence the source region exerted over the target region (see Table 3). It can be clearly seen that the slope of the plots of the PPI data acquired during the performance of the working memory task in quiet (that needs less attention) differs from that obtained from the performance of the working memory with babble noise as background (that needs more attention), except for the one shown in Fig. 9(a). The change in the slope of a plot when measured in a different context indicates a psychophysiologic interaction, with babble noise as contextual input modulating the influence of the activity in STG on the response of the target regions. It is very interesting to see that in the left hemisphere, the slope is steeper for the condition that needs more attention (working memory task in noisy background) as compared to the condition requiring less attention (working memory task in quiet). These involve the interactions of left TE1.1 vs. left STG, left BA3a vs. left STG and SMA vs. left STG. In contrast, in the right hemisphere, the slope for the condition requiring less attention (WMQ) is steeper than the slope for the condition requiring more attention (WMN), involving interactions of right SFG vs. right STG, SMA vs. right STG and right putamen vs. right STG. However, the interaction of right BA4p vs. right STG is similar in both WMQ and WMN conditions.

A steeper slope of a PPI plot means that a small change in activity in the STG results in a relatively larger response in the target regions as compared to a plot with less steeper slope. For TE1.1 and BA3a in the left hemisphere including the SMA, the interaction between these regions and STG is said to be mediated by babble noise and their responses to the activity in the left STG is increased in the presence of babble noise. TE1.1, together with TE1.0 and TE1.2 are known to be parts of the primary auditory cortex and are grouped together as TE1 by Morosan et al. (2001). They have tonotopic progressive characteristics such that the high frequencies are represented in TE1.1 while the lower frequencies are in TE1.2. The intermediate frequencies that are important for speech are processed in TE1.0. The TE1.1 region which is not directly involved with phonological working memory task shows an increase in the slope of the PPI plot in noise as compared to in quiet, indicating an increase in the effort in perceiving and processing the sound of the stimulus presented in a noisy background. The same argument is proposed to be valid for the SMA and BA3a. The SMA has the functions of retrieval of learned sequences that are under internal control such as the performance of a sequence of movements from memory. The fact that the SMA is activated even in the performance of phonological working memory task is possibly related to considerations of the diverse activities in which the SMA plays a role, suggesting that existing theories may not fully capture the fundamental functions of this region (Nachev et al. 2008). The significant activation observed in the left BA3a is due to the execution of action and speech when the participant was performing the task in which the participant is required to repeat backward the series of five words presented. The existence of SMA-STG and SMA-BA3a interactions supports the concept of audio-motor interaction (Hickok et al. 2003).

On the opposite hemisphere, the PPI plots of the response of the target regions as a function of the activity of the source region exhibited an increase in the slope of the regression in quiet as compared to in noise. This could be 
due to a direct involvement of the three areas, namely the superior frontal gyrus (SFG), putamen and SMA in the execution of the task, in response to the activity in the right STG. The SFG is also known to be located in the dorsolateral prefrontal cortex (DLPFC), classified as BA8 and BA9. This frontal area is involved in management of uncertainty in which increasing uncertainty increases activation. Uncertainty may exist during performance of the task due to the participant's inability to completely capture what is presented. This area is also known to be involved in sustaining attention and working memory (Brzezicka et al. 2011). Similar arguments apply to the putamen in which reinforcement and implicit learning, which are intrinsically part of the task, might explain its significant involvement. Apart from that, the putamen which is known to be interconnected to various pathways will certainly be activated due to its involvement in various regional interactions. As for the right BA4p, the phonological working memory task used required the participant to repeat words, resulting in significant activation in this area. The fact that the function of this area is more inclined towards motor coordination than working memory processing has rendered the PPI with the source region similar whether the task is performed in quiet or in noise. The fact that the SMA has shown a different response in PPI plots for the task presented in noise and in quiet when subjected to the activity in the left and right STG needs further investigation, especially when noise is used as the modulatory input.

\section{Conclusion}

In conclusion, the phonological working memory task based on the repeat backward test used in this study activates several brain regions that are involved in processing of the task. Similar brain regions are activated regardless of whether the task is performed in noise or in quiet. However, the activated brain regions in the longitudinal range of $70 \mathrm{~mm}$ to $20 \mathrm{~mm}$ appear to have extended activation when the task is performed in quiet due to the sensitivity of particular brain regions in perceiving the stimulus and later performing the task in quiet. Conjunction analysis reveals task-related areas which are the supplementary motor area (SMA), left TE1.1, left Brodmann Area (BA) 3a, right BA4p, right putamen and right superior frontal gyrus (SFG) which are activated with $p$ values well below 0.05 after controlling for family wise error (FWE). A steeper psychophysiologic interaction (PPI) slope for the right hemisphere task-related regions during the quiet condition indicates a larger influence of the source region (right STG) onto the target regions (SMA, right putamen, right SFG), except for BA4p which shows similar PPI slopes for in-quiet and in-noise measurements. For the left hemisphere regions (left TE1.1, left BA3a) and SMA, the interaction is modulated by noise, resulting in the increase in the PPI slope in the noisy condition. It can be said that the babble noise used in this study has little influence on the responses of working memory processes related to areas of input of the source region, resulting in a higher response of those areas in quiet as compared to in noise. This study, even though performed on a single participant has revealed several important fundamental insights for future multiple participant fMRI studies on working memory and is potentially viable to be implemented in a clinical environment. However, due to the different approaches used in studies of working memory which have employed various tasks and methodologies, we found that it is quite difficult for a direct comparison with the results obtained from other studies to be made at this time.

\section{Acknowledgements}

The authors would like to thank Sa'don Samian, the MRI Technologist of the Universiti Kebangsaan Malaysia Hospital (HUKM), for the assistance in the scanning and the Department of Radiology, Universiti Kebangsaan Malaysia Hospital for the permission to use the MRI scanner. The authors were also indebted to Dr. Stephan Kiebel and Dr. Carsten Müller from Max Planck Institute for Human Cognitive and Brain Sciences, Leipzig, Germany, for valuable discussions on experimental methods and data analyses. Parts of the Methods and Results sections of this article were written at the Max Planck Institute during a research attachment. This work was supported by the UKM-GUP-SK-07-20-205 research grant.

\section{References}

Abd Hamid, A. I., Yusoff, A. N., Mukari, S. Z., \& Mohamad, M. (2011). Brain Activation during Addition and Subtraction Tasks In-Noise and In-Quiet. Malays J Med Sci, 18(2), 3-15.

Amaro, E., Jr., \& Barker, G. J. (2006). Study design in fMRI: basic principles. Brain Cogn, 60(3), 220-232. http://dx.doi.org/10.1016/j.bandc.2005.11.009

Baddeley, A. D. (1996). Exploring the central executive. Quarterly J Exp Psychol, 49A, 5-28. http://dx.doi.org/10.1080/713755608

Baddeley, A. D. (2002). Is working memory still working? European Psychologist, 7(2), 85-97. http://dx.doi.org/Doi 10.1021//1016-9040.7.2.85 
Baddeley, A. D., \& Hitch, G. J. (1974). Working memory. In Recent advances in learning and motivation (Bower GA, ed.). Academic Press, New York, p. 47-90.

Belin, P., Zatorre, R. J., Hoge, R., Evans, A. C., \& Pike, B. (1999). Event-related fMRI of the auditory cortex. Neuroimage, 10(4), 417-429. http://dx.doi.org/10.1006/nimg.1999.0480

Bernal, B., \& Altman, N. R. (2001). Auditory functional MR imaging. AJR Am J Roentgenol, 176(4), 1009-1015. http://dx.doi.org/10.2214/ajr.176.4.1761009

Brzezicka, A., Sedek, G., Marchewka, A., Gola, M., Jednorog, K., Krolicki, L., \& Wrobel, A. (2011). A role for the right prefrontal and bilateral parietal cortex in four-term transitive reasoning: An fMRI study with abstract linear syllogism tasks. Acta Neurobiol Exp (Wars), 71(4), 479-495.

Eickhoff, S. B., Stephan, K. E., Mohlberg, H., Grefkes, C., Fink, G. R., Amunts, K., \& Zilles, K. (2005). A new SPM toolbox for combining probabilistic cytoarchitectonic maps and functional imaging data. Neuroimage, 25(4), 1325-1335. http://dx.doi.org/10.1016/j.neuroimage.2004.12.034

Friston, K. J., Buechel, C., Fink, G. R., Morris, J., Rolls, E., \& Dolan, R. J. (1997). Psychophysiological and

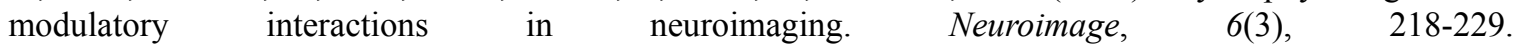
http://dx.doi.org/10.1006/nimg.1997.0291

Friston, K. J., Holmes, A., Poline, J. B., Price, C. J., \& Frith, C. D. (1996). Detecting activations in PET and fMRI: levels of inference and power. Neuroimage, 4(3Pt1), 223-235. http://dx.doi.org/10.1006/nimg.1996.0074

Friston, K. J., Mechelli, A., Turner, R., \& Price, C. J. (2000). Nonlinear responses in fMRI: The Balloon model, Volterra kernels, and other hemodynamics. Neuroimage, 12(4), 466-477. http://dx.doi.org/10.1006/nimg.2000.0630

Gaab, N., Gabrieli, J. D., \& Glover, G. H. (2007). Assessing the influence of scanner background noise on auditory processing. II. An fMRI study comparing auditory processing in the absence and presence of recorded scanner noise using a sparse design. Hum Brain Mapp, 28(8), 721-732. http://dx.doi.org/10.1002/hbm.20299

Gaab, N., Gaser, C., Zaehle, T., Jancke, L., \& Schlaug, G. (2003). Functional anatomy of pitch memory--an fMRI study with sparse temporal sampling. Neuroimage, 19(4), 1417-1426. http://dx.doi.org/10.1016/S1053-8119(03)00224-6

Gathercole, S. E. (1999). Cognitive approaches to the development of short-term memory. Trends Cogn Sci, 3(11), 410-419. http://dx.doi.org/10.1016/S1364-6613(99)01388-1

Hall, D. A., Haggard, M. P., Akeroyd, M. A., Palmer, A. R., Summerfield, A. Q., Elliott, M. R., Gurney, E. M., \& Bowtell, R. W. (1999). "Sparse" temporal sampling in auditory fMRI. Hum Brain Mapp, 7(3), 213-223. http://dx.doi.org/10.1002/(SICI)1097-0193(1999)7:3<213::AID-HBM5>3.0.CO;2-N

Hall, D. A., Summerfield, A. Q., Goncalves, M. S., Foster, J. R., Palmer, A. R., \& Bowtell, R. W. (2000). Time-course of the auditory BOLD response to scanner noise. Magn Reson Med, 43(4), 601-606. http://dx.doi.org/10.1002/(SICI)1522-2594(200004)43:4<601::AID-MRM16>3.0.CO;2-R

Hickok, G., Buchsbaum, B., Humphries, C., \& Muftuler, T. (2003). Auditory-motor interaction revealed by fMRI: Speech, music, and working memory in area Spt. $J$ Cogn Neurosci, 15(5), 673-682. http://dx.doi.org/10.1162/089892903322307393

Hwang, J. H., Wu, C. W., Chou, P. H., Liu, T. C., \& Chen, J. H. (2005). Hemispheric difference in activation patterns of human auditory-associated cortex: An FMRI study. ORL J Otorhinolaryngol Relat Spec, 67(4), 242-246. http://dx.doi.org/10.1159/000089501

Jancke, L., Peters, M., Schlaug, G., Posse, S., Steinmetz, H., \& Muller-Gartner, H. (1998). Differential magnetic resonance signal change in human sensorimotor cortex to finger movements of different rate of the dominant and subdominant hand. Brain Res Cogn Brain Res, 6(4), 279-284. http://dx.doi.org/10.1016/S0926-6410(98)00003-2

Karlsgodt, K. H., Shirinyan, D., van Erp, T. G., Cohen, M. S., \& Cannon, T. D. (2005). Hippocampal activations during encoding and retrieval in a verbal working memory paradigm. Neuroimage, 25(4), 1224-1231.

Langers, D. R., \& van Dijk, P. (2012). Mapping the tonotopic organization in human auditory cortex with minimally salient acoustic stimulation. Cereb Cortex, 22(9), 2024-2038. http://dx.doi.org/10.1093/cercor/bhr282 
Langers, D. R., Backes, W. H., \& van Dijk, P. (2007). Representation of lateralization and tonotopy in primary versus secondary human auditory cortex. Neuroimage, 34(1), 264-273. http://dx.doi.org/10.1016/j.neuroimage.2006.09.002

Lutz, K., Koeneke, S., Wustenberg, T., \& Jancke, L. (2005). Asymmetry of cortical activation during maximum and convenient tapping speed. Neurosci Lett, http://dx.doi.org/S0304-3940(04)01220-010.1016/j.neulet.2004.09.058

Mueller, K., Mildner, T., Fritz, T., Lepsien, J., Schwarzbauer, C., Schroeter, M. L., \& Moller, H. E. (2011). Investigating brain response to music: a comparison of different $\mathrm{fMRI}$ acquisition schemes. Neuroimage, 54(1), 337-343. http://dx.doi.org/10.1016/j.neuroimage.2010.08.029

Nachev, P., Kennard, C., \& Husain, M. (2008). Functional role of the supplementary and pre-supplementary motor areas. Nature Reviews Neuroscience, 9(11), 856-869. http://dx.doi.org/10.1038/nrn2478

Oldfield, R. C. (1971). The assessment and analysis of handedness: The Edinburgh inventory. Neuropsychologia, 9(1), 97-113. http://dx.doi.org/10.1016/0028-3932(71)90067-4

Specht, K., \& Reul, J. (2003). Functional segregation of the temporal lobes into highly differentiated subsystems for auditory perception: An auditory rapid event-related fMRI-task. Neuroimage, 20(4), 1944-1954. http://dx.doi.org/10.1016/j.neuroimage.2003.07.034

Toepper, M., Markowitsch, H. J., Gebhardt, H., Beblo, T., Thomas, C., Gallhofer, B., Driessen, M., \& Sammer, G. (2010). Hippocampal involvement in working memory encoding of changing locations: An fMRI study. Brain Res, 1354, 91-99. http://dx.doi.org/10.1016/j.brainres.2010.07.065

Wong, P. C., Jin, J. X., Gunasekera, G. M., Abel, R., Lee, E. R., \& Dhar, S. (2009). Aging and cortical mechanisms of speech perception in noise. Neuropsychologia, 47(3), 693-703. http://dx.doi.org/10.1016/j.neuropsychologia.2008.11.032

Wong, P. C., Uppunda, A. K., Parrish, T. B., \& Dhar, S. (2008). Cortical mechanisms of speech perception in noise. J Speech Lang Hear Res, 51(4), 1026-1041. http://dx.doi.org/10.1044/1092-4388(2008/075)

Yusoff, A. N., Mohamad, M., Hamid, K. A., Abd Hamid, A. I., \& Mukari, S. Z. M. S. (2011). Acquisition, Analyses and Interpretation of fMRI Data: A Study on the Effective Connectivity in Human Primary Auditory Cortices. Sains Malaysiana, 40(6), 665-678.

\section{Copyrights}

Copyright for this article is retained by the author(s), with first publication rights granted to the journal.

This is an open-access article distributed under the terms and conditions of the Creative Commons Attribution license (http://creativecommons.org/licenses/by/3.0/). 